\title{
Primary Penile Cancer + Peyronie's Disease $=$ Diagnostic Difficulty: A Case of Delayed Diagnosis with a Review of the Problem of Penile Neoplasms Masquerading as, or Being Masked by, Peyronie's Disease
}

\author{
Chris Hurrell ${ }^{1}$, Stuart Irving ${ }^{1}$, Melanie Shaw ${ }^{2, \S}$, Richard Y. Ball ${ }^{3}$ and Sudhanshu Chitale ${ }^{*}, 1$ \\ Departments of ${ }^{1}$ Urology, ${ }^{2}$ Radiology \& ${ }^{3}$ Histopathology, Norfolk \& Norwich University Hospitals NHS Foundation \\ Trust, Norwich, NR4 7UY, UK
}

\begin{abstract}
Squamous cell carcinoma of the penis is a rare malignant neoplasm presenting as a hard painless lump, usually on the glans or prepuce but also, rarely, on the shaft. Peyronie's disease is a relatively common (prevalence about 3\%) benign condition that presents as a penile plaque or flat lump with or without penile curvature or deformity. We report a case of squamous cell carcinoma of the penile shaft masquerading as, or being masked by, Peyronie's disease.
\end{abstract}

Keywords: Peyronie's disease, carcinoma, penis.

\section{CASE REPORT}

An 86-year-old man presented with a flat, disc-like, painless plaque in the subcutaneous plane along the penile shaft and inseparable from the tunica albuginea. According to the patient, it had been unchanged for several years. It was situated on the dorsal aspect of the mid-shaft of the penis, clinically also involving the inter-corporal septum. The lump ( $5 \mathrm{~cm}$ long $\times 2 \mathrm{~cm}$ wide) was hard, irregular, and non-tender. No signs of involvement of the penile skin were recognised (i.e., there was no ulceration, excoriation, bleeding, discharge, phimosis or any other skin lesion). The glans appeared entirely normal, with no features to suggest carcinoma in situ. However, there was evidence of mild, painless chronic paraphimosis on the ventral aspect along the coronal sulcus, but not covering any aspect of the glans penis. Groin lymph nodes were unremarkable. Benign enlargement of his prostate was noted on digital rectal examination.

His past history included hypertension, hypothyroidism, chronic renal disease, abdominal aortic aneurysm, and a posterior circulation stroke in 2005. In addition to stable lower urinary tract symptoms, he also admitted to longstanding erectile dysfunction and was consequently unable to comment upon (and seemed unconcerned about) any penile curvature or deformity on erection. A clinical diagnosis of Peyronie's plaque/disease was made. Bearing in mind the patient's age, lack of symptoms and his specific request; non-surgical (conservative) management was agreed upon, and no further invasive investigations/procedures, such as corrective penile surgery or incisional biopsy or even MRI scanning, were planned or undertaken. No medical/drug therapy was offered for the penile plaque as the patient had no bothersome symptoms related to it.

*Address correspondence to this author at the Department of Urology, Norfolk \& Norwich University Hospital, Colney Lane, Norwich, NR4 7UY, England; Tel: 0044 7748944385; E-mail: chitalenorwich@aol.com

${ }^{\S}$ Dr Shaw died on 9 July 2011.
Eighteen months later, the patient represented with macroscopic haematuria and co-existent pneumonia. Genital examination revealed the pre-existent hard lump on the penile shaft with persistent paraphimosis and, on this occasion, a suspect, erythematous, flat lesion on the glans penis. Ultrasound imaging of the urinary tract was unremarkable and flexible cystourethroscopy revealed induration and erythema of the distal/anterior urethra but no urothelial lesion in the urethra or bladder.

An MRI scan, however, showed abnormal texture and signal in the entire penis, implying diffuse corporal infiltration throughout the organ (Fig. 1), with multiple

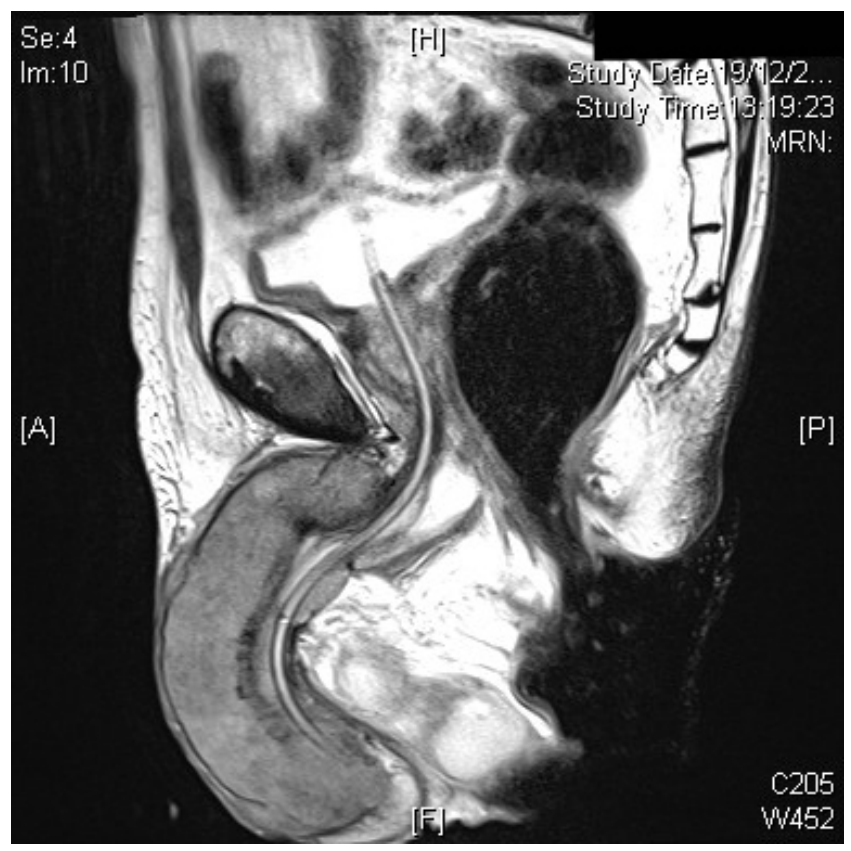

Fig. (1). MRI scan of the penis showing diffuse corporal infiltration by the tumour. 
involved pelvic nodes (including the left obturator, left iliac, and bilateral inguinal groups) and bone metastases (rT4, N2, M1). Abnormal soft tissue replaced the inferior left pubic ramus and the anterior aspect of the inferior right pubic ramus. There were metastatic deposits in both the right and left acetabula, with possible early protrusio acetabulae on the right. The penis was clearly the primary site/origin of the tumour, and there was no clinical or radiological evidence that it was metastatic from a different site. Chest X-ray showed no evidence of neoplasia. Regrettably, a clinical photograph was not obtained at initial presentation or subsequently.

Three incisional wedge biopsies of the penile lesion were examined. They comprised four pieces of tissue, from the shaft, corpora and glans, ranging in size from $7 \times 5 \times 4 \mathrm{~mm}$ to $15 \times 10 \times 10 \mathrm{~mm}$. The sections all showed conventional invasive squamous cell carcinoma (up to grade 3 ) of the penis (Fig. 2a) with widespread necrosis and vascular and perineural permeation. The section from the glans penis included some normal epidermis and also areas of squamous cell carcinoma in situ (Fig. 2b), confirming that the carcinoma had arisen locally.

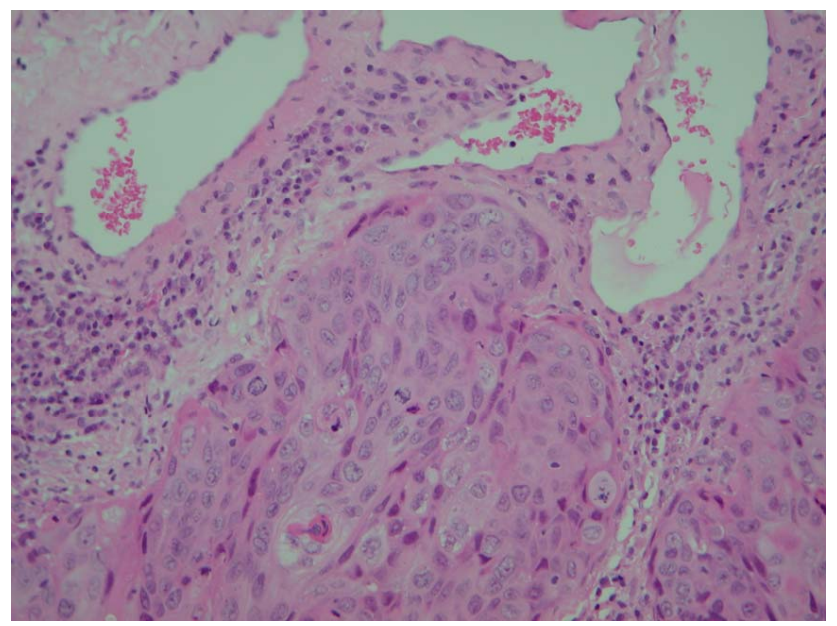

Fig. (2a). Histopathological section showing poorly differentiated invasive squamous cell carcinoma of the penis $(\mathrm{H} \& \mathrm{E} \times 20$ objective).

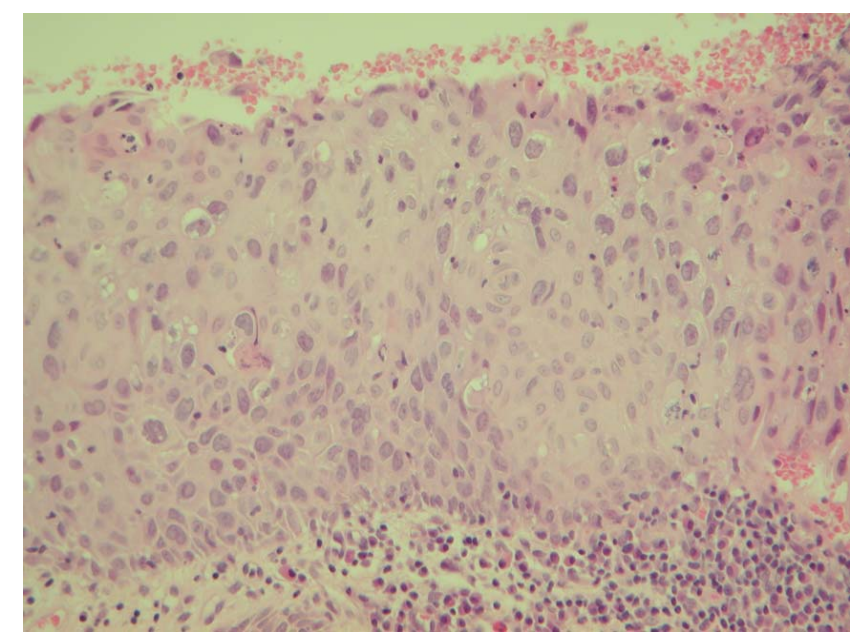

Fig. (2b). Histopathological section showing squamous cell carcinoma in situ of the glans penis (H \& E x 20 objective).
Palliative/supportive care was offered in view of the extent of the disease at diagnosis and the overall poor prognosis. The patient died in hospital two months later of a combination of heart failure, renal failure, and pneumonia. The patient's relatives did not consent to clinical autopsy.

\section{DISCUSSION \& REVIEW OF LITERATURE}

Peyronie's disease is a benign condition in which a fibrous plaque forms in the tunica albuginea of the penis, resulting in a palpable mass and increased curvature of the penile shaft. The plaque most commonly presents on the dorsal surface of the penis and may be painful in the acute phase. Prevalence is approximately $1-3 \%$ [1], with an age of onset usually between 40-60 years.

Squamous cell carcinoma of the penis is a rare malignancy with incidence rates of $0.3-8 \times 10^{-5}$ [2]. It accounts for about $95 \%$ of penile cancers. It generally presents as a hard painless lump, usually on the glans or prepuce, with fewer than $2 \%$ of cases presenting with a lump on the shaft [3]. Various types of squamous cell carcinoma are described in the WHO classification scheme [4]. Like this patient, most cases are conventional in histopathological appearance (ICD-O code 8070/3) and diagnosis is readily achieved without recourse to ancillary studies such as immunohistochemical staining.

This case highlights a rare presentation of a squamous cell carcinoma of the penis in a man who seemed to have had Peyronie's disease for several years. Given its advanced stage when diagnosed, the carcinoma may have been masked by the benign plaque, with consequent diagnostic difficulty and delay. We cannot be sure, but it is likely that the carcinoma was already developing when he first presented with penile disease. This patient reminds us that it is important to consider and exclude rarer, but more serious, conditions in the initial differential diagnostic assessment of penile disease. The presence of a painless chronic paraphimosis should alert the clinician to the possibility of lymphoedema secondary to a proximal penile neoplastic lesion and warrant timely further investigations to rule out sinister pathology.

Various primary penile neoplasms, usually malignant, have been described as mimicking Peyronie's disease in their presentation (Table 1). They include primary B-cell nonHodgkin lymphoma [5], angiosarcoma [6], atypical epithelioid haemangioma [7], and epithelioid sarcoma [8 12]. Advanced caecal carcinoma and penile metastases [4] from genito-urinary malignancies may also present in a similar manner with retrograde lymphatic and venous spread as the most likely underlying mechanism.

Epithelioid sarcoma of the penis, a rare malignant condition, has most often been reported as mimicking Peyronie's disease. Thirteen cases of penile epithelioid sarcoma, four masquerading as Peyronie's disease [8 -11], were reported in the literature up to 2003; to our knowledge, no further reports have appeared. Age is an important clue in the differential diagnosis between penile epithelioid sarcoma and Peyronie's disease. Patients with penile epithelioid sarcoma tend to be relatively young (age range $=23-43$ years; mean $=34$ years; $[9,10]$ compared with those with Peyronie's disease. However, other types of malignant disease may present as Peyronie's disease at an older age 
(Table 1), appropriate for the type of neoplasm. Symptoms of urethral obstruction, persistently painful and increasing size of the nodule/lump, paraphimosis, and haematuria are also important clues to the diagnosis of malignant disease (Table 1) and should prompt biopsy or other investigation.

Synchronous or metachronous metastasis to the penis is uncommon but should be considered in the differential diagnosis of painless discrete penile lumps. MRI features of such metastatic, as well as of primary, penile lesions are characteristic. Metastases typically show multiple discrete masses within the penile corpora. There was no evidence in this patient to suggest that his penile cancer was metastatic, and there was no history of previous malignant disease. Indeed, histopathological evidence of squamous cell carcinoma in situ on penile biopsy supported a local origin. His relatively long survival without specific anti-tumour therapy, both after initial presentation and later definitive diagnosis, would also make metastatic disease unlikely; the prognosis of secondary tumours of the penis is usually very poor, often measured in weeks [4].
Because malignant tumours presenting as or being masked by Peyronie's disease are rare, there may be a delay in reaching the correct diagnosis.. The duration of symptoms in patients with penile neoplasms mimicking Peyronie's disease before a diagnosis was achieved ranged from 5 months to more than 13 years (Table 1). The delay seems to be related to slow growth and harmless appearance of the lesion. In some cases, histopathological misdiagnosis may have contributed to the delay in diagnosis $[8,10]$. In others, earlier diagnoses were reassuring and may have been correct; the reports do not give unequivocal evidence of error [5, 9].

Ultrasonography and radiography have not been consistent in the reliable diagnosis of Peyronie's disease, but MRI has been shown to have a definite role in evaluation of pathological processes involving the penis [9, 10]. MRI examination of all patients presenting as Peyronie's disease would not be judicious because of the rarity of malignant disease presenting in that manner. There is a role for Positron Emission Tomography (PET) and CT scanning as a whole body screen to locate the primary tumour/distant

Table 1.

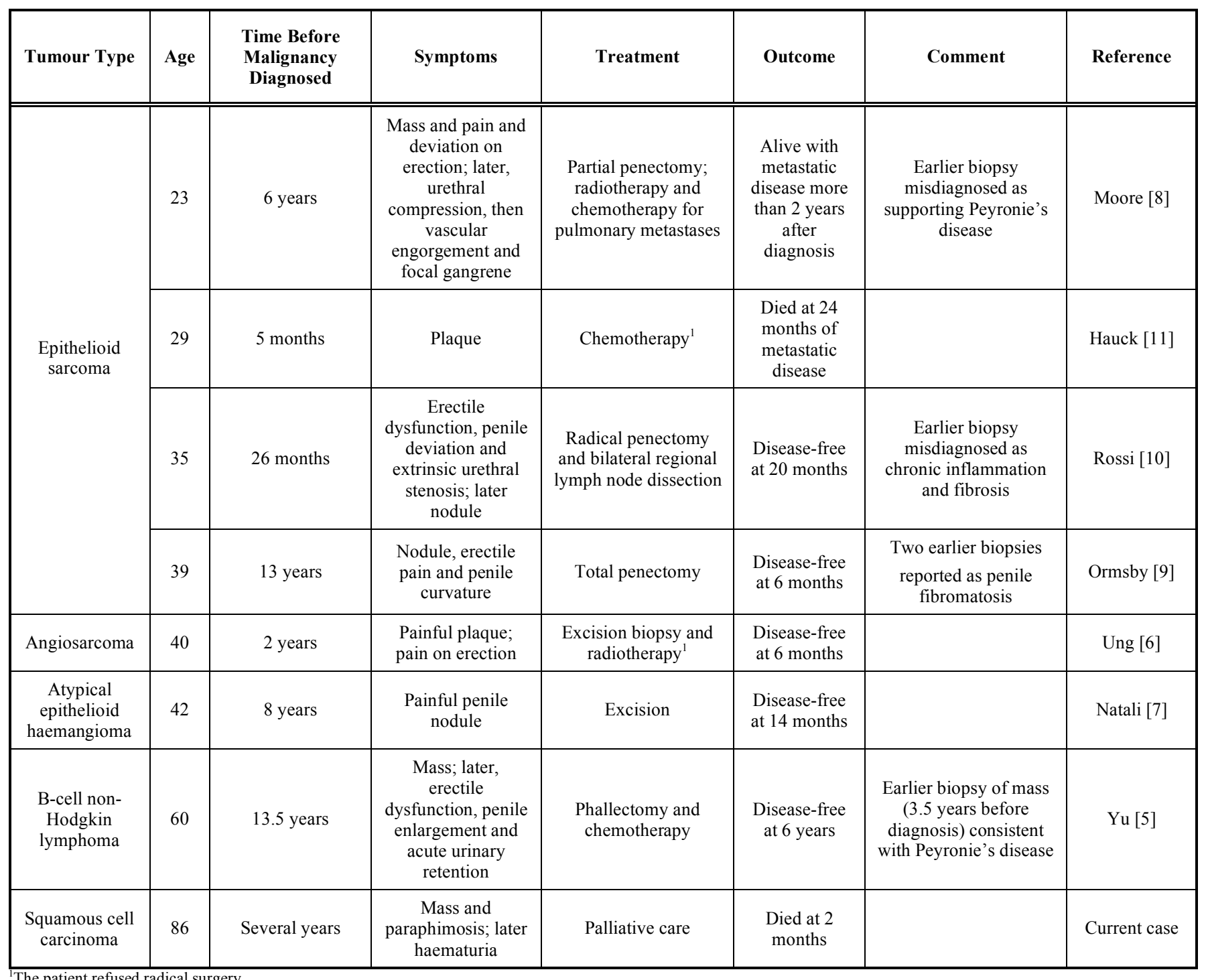

The patient refused radical surgery. 
metastases. Abnormal clinical features, including young age and those noted in the table, should raise the question of further investigation, including MRI and biopsy. In such cases, the radiologist and histopathologist need to be alerted to the possibility of malignancy masquerading as Peyronie's disease. In patients presenting with probable Peyronie's plaque/lump who opt for expectant management/observation, it is important that they are warned to report any significant change in their "lesion" as that would entail an urgent review before the routine 3- to 6-monthly follow up.

This case suggests that urologists should have a high index of suspicion and low threshold for investigating painless penile plaques/lumps that simulate Peyronie's disease, particularly in the elderly. In these patients, timely MRI followed by corporal wedge biopsy might help reach an early diagnosis and offer potentially curative treatment; such management should be considered earlier than is often the case, especially in older men with an atypical presentation. As neoplastic penile lesions are rare, whilst Peyronie's disease is relatively much more common, we would not recommend over-investigating these patients by unnecessary invasive investigations. Instead, we advocate early referral to a tertiary centre or consulting a specialist with expertise in Peyronie's disease before subjecting them to an appropriate test for their suspected penile lesion.

\section{ACKNOWLEDGEMENT}

Declared none.

\section{CONFLICT OF INTEREST}

The authors confirm that this article content has no conflict of interest.

\section{REFERENCES}

[1] Ralph D, Gonzalez-Cadavid N, Mirone V, et al. The management of Peyronie's disease: evidence-based 2010 guidelines. J Sex Med 2010; 7: 2359-74.

[2] Vatanasapt V, Martin N, Sriplung MH, et al. Cancer incidence in Thailand, 1988-1991. Cancer Epidemiol Biomarkers Prev 1995; 4: 475-83.

[3] Sufrin G, Huben R. Benign and malignant lesions of the penis. In: Gillenwater JY, Ed. Adult and Pediatric Urology, $2^{\text {nd }}$ ed. Chicago: Year Book 1991; pp. 1997-2042.

[4] Eble JN, Sauter G, Epstein JI, Sesterhenn IA (Eds.). Chapter 5, Tumours of the penis, in Pathology and genetics of tumours of the urinary system and male genital organs. World Health Classification of Tumours. Lyon: IARC Press 2004; pp. 279-98.

[5] Yu GS, Nseyo UO, Carson JW. Primary penile lymphoma in a patient with Peyronie's disease. J Urol 1989; 142: 1076-7.

[6] Ung JO, Padera RF, O'Leary MP. Angiosarcoma of the penis masquerading as a Peyronie's plaque. J Urol 2002; 167: 1785-6.

[7] Natali A, Nesi G, Vittori G, et al. Rare case of atypical epithelioid hemangioma of penis initially diagnosed as Peyronie's disease: report with clinical, radiologic, and immunohistochemical analysis. Urology 2009; 73: 210.e7-10.

[8] Moore SW, Wheeler JE, Hefter LG. Epithelioid sarcoma masquerading as Peyronie's disease. Cancer 1975; 35: 1706-10.

[9] Ormsby AH, Liou LS, Oriba HA, Angermeier KW, Goldblum JR. Epithelioid sarcoma of he penis; report of an unusual case and review of the literature. Ann Diagn Pathol 2000; 4: 88-94.

[10] Rossi G, Ferrari G, Longo L, Trentini GP. Epithelioid sarcoma of the penis: a case report and review of the literature. Pathol Int 2000; 50: 579-85.

[11] Hauck EW, Schmelz HU, Diemer T, et al. Epithelioid sarcoma of the penis - a rare differential diagnosis of Peyronie's disease. Int J Impot Res 2003; 15: 378-82.

[12] Usta MF, Adams DM, Zhang JW, Davis R, Hellstrom WJ. Penile epithelioid sarcoma and the case for a histopathological diagnosis in Peyronie's disease. BJU Int 2003; 91: 519-21. 